\title{
Mapeamento e análise de desempenho da graduação e da pós-graduação em Engenharia de Produção no Brasil
}

\section{Mapping and analysis of undergraduate and graduate Production Engineering courses in Brazil}

\author{
Carla Hartmann Sturm 1 \\ Patrícia Schrippe ${ }^{2}$ \\ Flaviani Souto Bolzan Medeiros ${ }^{2}$ \\ Jaíne Fátima Koschek ${ }^{1}$ \\ Andreas Dittmar Weise ${ }^{2}$
}

\begin{abstract}
Resumo: A universidade se afirma como instituição por excelência, em todas as suas dimensões, para o cultivo do saber num constante processo de construção e reconstrução, em busca do desenvolvimento da cultura e pensamento. Nesse sentido, este artigo tem como objetivo analisar os cursos brasileiros de graduação e de pós-graduação em Engenharia de Produção, considerando a ênfase do curso, as áreas de pesquisa, a situação geográfica e a sua classificação. Para isso, desenvolveu-se um estudo qualitativo e quantitativo, do tipo descritivo, tendo como plano de coleta dos dados uma pesquisa bibliográfica a partir dos dados disponibilizados nos sites da Associação Brasileira de Engenharia de Produção (ABEPRO) e do Ministério da Educação (e-MEC). O estudo aponta que, apesar do curso de graduação e de pós em Engenharia de Produção terem se desenvolvido no país, a sua distribuição é heterogênea e a qualidade se encontra aquém das necessidades do mercado.
\end{abstract}

Palavras-chave: Graduação em Engenharia de Produção. Pós-graduação em Engenharia de Produção. Mapeamento. Ensino em Engenharia de Produção.

\begin{abstract}
The University is consolidated as an institution of excellence in all its dimensions, by the cultivation of knowledge in a constant process of construction and reconstruction aiming at the development of culture and thought. Thus, this paper aims to analyze the Brazilian undergraduate and graduate courses in Production Engineering, considering their emphasis, research areas, geographical location, and classification. To this end, a qualitative, quantitative and descriptive study was developed, with a plan to collect information through a literature search of the data available on the websites of the Brazilian Association of Industrial Engineering (ABEPRO) and the Ministry of Education (e-MEC). The study indicates that, although the undergraduate and graduate courses in Production Engineering have improved in the country, their distribution is heterogeneous and their quality falls short of the market's requirements.
\end{abstract}

Keywords: Undergraduate courses in Production Engineering. Graduate courses in Production Engineering. Production Engineering. Mapping. Production Engineering Education.

\section{Introdução}

Nas últimas décadas houve um período turbulento de mudanças sociais, tecnológicas, econômicas, culturais e políticas. O fenômeno da globalização da economia, por sua vez, sacudiu as universidades e lançou alguns novos desafios. Assim, pode-se dizer que as instituições de ensino superior só estarão aptas para sobreviver no meio educacional se

tiverem qualidade e operarem de forma competitiva (FOWLER et al., 2011). A crescente importância do conhecimento e a valorização do capital intelectual dos indivíduos, bem como a revolução da informação e dos meios de comunicação passaram a ser permanentemente discutidas, inclusive em fóruns internacionais.

\footnotetext{
${ }^{1}$ Universidade Federal de Santa Maria - UFSM, CEP 97105-900, Santa Maria, RGS, Brasil, e-mail: carlasturm@hotmail.com; jah. koschek@hotmail.com

${ }^{2}$ Programa de Pós-graduação em Engenharia de Produção, Universidade Federal de Santa Maria - UFSM, CEP 97105-900, Santa Maria, RS, Brasil, e-mail: pschrippe@gmail.com;flaviani.13@gmail.com; mail@weise.de
}

Recebido em Set. 5, 2013 - Aceito em Out. 1, 2014

Suporte Financeiro: Nenhum. 
Na concepção de Stallivieri (2004), esses desafios impelem as universidades a revisar e atualizar as suas estratégias a fim de que seus estudantes e egressos passem a contar com as competências essenciais, acadêmicas e profissionais que lhes permitam interagir numa sociedade cada vez mais multicultural, com rápidas mudanças em seus sistemas.

Segundo a Folha de S. Paulo (FOLHA..., 2013), profissionais das áreas de Engenharia Mecânica, Engenharia de Produção e Engenharia Civil estão entre as profissões em maior falta no mercado nacional. A tentativa das empresas, visando amenizar esses problemas, é reduzir as exigências no momento da contratação.

Nesse sentido, Chryssolouris et al. (2013) complementam que a globalização e expansão tecnológica geram e tendem a ampliar as exigências de habilidades, portanto, uma adaptação no sentido de ampliar a educação da engenharia integrada ao desenvolvimento de profundo e estruturado conhecimento de multicompetências se faz necessário.

Concomitantemente às competências técnicas relacionadas à produção e controle, o mercado, de acordo com Conceição et al. (2012), exige competências como liderança, eficiência na comunicação oral e escrita, espírito empreendedor, fluência em mais de uma língua estrangeira, dentre outras. Nessa conjuntura, os ingressantes no ensino superior nacional anteferem cursos mais versáteis, como as tradicionais Engenharia Civil e Engenharia Mecânica e, nos últimos anos, também nas áreas de Produção e Elétrica (INSTITUTO..., 2011).

No caso, o engenheiro de produção, segundo o Ministério da Educação (BRASIL, 2014), é um profissional de formação generalista que, entre outras funções, projeta, implanta, opera e otimiza e, também, mantém sistemas integrados de produção tanto de bens como de serviços e, ainda, coordena e supervisiona equipes de trabalho.

Com base nesse contexto, este artigo tem como objetivo analisar os cursos brasileiros de graduação e de pós-graduação em Engenharia de Produção considerando, especialmente, a sua situação geográfica e classificação. Este estudo justifica-se considerando o fato das rápidas e profundas mudanças que vêm ocorrendo no mundo contemporâneo, e no campo da educação isso não é diferente (BENFATTI \& STANO, 2010).

A universidade como instituição que cultiva o trabalho da razão e formação humana se institui e se autojustifica à medida que faz a crítica de si mesma, da sociedade, da cultura, da existência humana, dos saberes, das ideias, dos conceitos, das teorias, dos métodos e da prática (PASSOS et al., 2005). Moreira $\&$ Veit (2010) corroboram, enfatizando que o ensino universitário visa a formação de profissionais éticos e competentes para atuação no mercado de trabalho, majoritariamente fora da universidade.

O presente trabalho encontra-se estruturado em seis seções: logo após a introdução, a seção 2 contempla o referencial teórico que embasou o estudo, incluindo tópicos como a universidade, o ensino e a pesquisa, como também breves discussões acerca da Engenharia de Produção e da evolução desses cursos. Em seguida, na seção 3, apresenta-se a metodologia utilizada para a realização da pesquisa, com suas respectivas classificações e etapas adotadas. Na sequência, na seção 4, constam o mapeamento dos cursos de graduação e de pós-graduação e, ainda, as linhas de pesquisa na pós-graduação em Engenharia de Produção. Na seção 5 segue a análise estatística dos cursos de graduação e de pós-graduação em Engenharia de Produção. O último item, a seção 6 , traz as considerações finais a partir do estudo realizado.

\section{Referencial teórico}

\subsection{A universidade, o ensino e a pesquisa}

Conforme Passos et al. (2005), as universidades nasceram na Idade Média, no contexto intenso de transformações na esfera da produção, da economia, da vida social, da cultura que se concretizava, sobretudo, nas cidades. $\mathrm{O}$ termo latino universitas significava na Roma antiga a universalidade, a totalidade, o todo, o universo, conjunto das coisas, o colégio, a associação ou a comunidade de pessoas. No século XII esse termo era empregado para se referir ao conjunto de pessoas que numa cidade exerciam o mesmo ofício e tinham, pois, interesses comuns, ou seja, uma corporação de ofício (PASSOS et al., 2005).

De acordo com Rocha (1997), a primeira vez que se empregou a expressão "universidade" num texto constitucional brasileiro foi em 1988, em todas as demais constituições o assunto sempre foi tratado mediante o uso de expressões como "ensino ulterior primário" ou "ensino superior".

Na sociedade brasileira, Velloso (2003) esclarece que a emergência do sistema de ensino superior constitui um acontecimento tardio. Ao contrário de alguns países da América Hispânica, o Brasil não possuiu nem instituições de ensino superior nem universidades durante o período colonial.

As primeiras escolas superiores no país somente foram criadas no início do século XIX, com a vinda da família real portuguesa para a colônia. Essas instituições tinham o claro objetivo de formar profissionais nas áreas de Medicina, 
Engenharia e Direito para desempenhar diferentes funções ocupacionais na Corte e encontravam-se sob o controle do Estado (VELLOSO, 2003, p. 55).

A pesquisa, enquanto função básica da universidade, insere-se dentro do contexto global da universidade, do conteúdo e forma de suas interações com a sociedade, decorrentes da autonomia universitária. O objetivo fundamental da universidade é pensar. Assim, o que justifica a universidade é a busca e o cultivo do saber, a formação de seres humanos que a todo o momento possam inserir-se de modo crítico, rigoroso e criativo na existência social, no mundo do trabalho, e contribuir para transformá-lo, para superar a realidade e a sociedade existente (DÍAZ BORDENAVE \& PEREIRA, 2012).

Sob essa perspectiva, Ferraz \& Belhot (2010, p. 421) acrescentam que

na educação, decidir e definir os objetivos de aprendizagem significa estruturar, de forma consciente, o processo educacional de modo a oportunizar mudanças de pensamentos, ações e condutas.

Portanto, o ensino com pesquisa leva o aluno à aquisição dos processos e procedimentos de produção de conhecimentos e não apenas aos produtos dessa processualidade. Dessa forma, pode-se dizer que, pela pesquisa, a universidade cria o saber e, pelo ensino, o saber se organiza e se difunde. A universidade se faz ela mesma à medida que responde, academicamente, aos desafios que a humanidade, as nações, os grupos e as pessoas lhe apresentam e afirma-se concretamente como instituição por excelência da cultura, da crítica, do pensamento, do trabalho intelectual, da formação humana, da criação e da invenção de novas ideias e formas de existência (LOPES et al., 2003).

Assim sendo, conforme Souza (2001), a educação superior tem como fim a criação, o cultivo, a difusão e a aplicação de conhecimento complexo e sistêmico dentre os elaborados pela humanidade ao longo dos tempos e é ministrada no Brasil em instituições catalogadas como universidades, centros universitários, faculdades integradas, faculdades e instituições superiores ou escolas superiores, conforme o artigo $12^{\circ}$ do decreto federal n. 5.773/06 (BRASIL, 2006).

Atualmente, ao contrário do início do século XIX, período em que o propósito era investir na formação de profissionais nas áreas de Medicina, Engenharia e Direito, com a expansão das universidades e incentivos do governo, percebe-se uma grande variedade de cursos oferecidos pelas instituições de ensino no país.

\subsection{Engenharia de Produção}

A Engenharia de Produção, de acordo com análises de Naveiro (2000), é a menos tecnológica dentre as engenharias, na medida em que é mais abrangente e genérica. $\mathrm{O}$ mesmo autor complementa que o engenheiro de produção é o único profissional do mercado com capacidade de enxergar os problemas de forma global, visto que conhece a realidade industrial e tecnológica, ainda que frequentemente não disponha de mecanismos para solucionar detalhes dos problemas.

A Engenharia de Produção trata do projeto, aperfeiçoamento e implantação de sistemas integrados de pessoas, materiais, informações, equipamentos e energia para a produção de bens e serviços, de maneira econômica, respeitando os preceitos éticos e culturais (FLEURY, 2008, p. 1).

Os profissionais de Engenharia de Produção buscam por meio de métodos de engenharia e de gerenciamento a otimização dos sistemas produtivos, no intuito de gerar o maior lucro e da maneira mais sustentável. A capacidade de atuação do engenheiro de produção de acordo com Weise \& Trierweiller (2010) ultrapassa os limites da área industrial, berço de sua formação, atingindo setores de serviço nos mais diversificados ramos. Dessa forma, a Engenharia de Produção faz uso de uma abordagem sistêmica, transitando entre aspectos inter, pluri e, inclusive, transdisciplinares, dependendo da complexidade dos aspectos envolvidos no problema em análise (IAROZINSKI NETO \& LEITE, 2010).

Assim como na graduação, o mestrado e o doutorado em Engenharia de Produção se dedicam a abranger diversos conteúdos para desenvolver, implementar, gerenciar, melhorar e/ou otimizar bens e serviços. O conhecimento acerca da Engenharia de Produção, que balizam Graduação, Pós-graduação e demais atividades, de acordo com ABEPRO (ASSOCIAÇÃO..., 2008), encontra-se relacionado nas seguintes áreas:

1) Engenharia de Operações e Processos da Produção: Âmbito relacionado a projetos, operações que criam e/ou entregam produtos da empresa;

2) Logística: Relacionado ao transporte, movimentação, estoque e armazenamento de insumos e produtos;

3) Pesquisa Operacional: Modelagem matemática aplicada, principalmente via softwares específicos;

4) Engenharia da Qualidade: Planeja, projeta e controla sistemas de gestão de qualidade fazendo uso de ferramentas específicas; 
5) Engenharia do Produto: Acervo de ferramentas e processos no intuito de desenvolver produtos;

6) Engenharia Organizacional: Refere-se a assuntos acerca da estratégia organizacional, inclui aspectos relacionados à inovação, tecnologia e desempenho organizacional;

7) Engenharia Econômica: Via projeções, fornece parâmetros quantitativos referentes às alternativas no intuito de auxiliar na tomada de decisão;

8) Engenharia do Trabalho: Estuda as interações entre pessoas e demais elementos do sistema produtivo;

9) Engenharia da Sustentabilidade: Planeja a utilização eficiente dos recursos naturais, atentando à responsabilidade social; $\mathrm{e}$

10) Educação em Engenharia de Produção: Reflete assuntos relacionados ao desenvolvimento e inserção da educação superior da Engenharia de Produção.

Nesse sentido, a Engenharia de Produção apresenta um leque de atuação bastante extenso e complexo, sendo requerido do profissional um amplo conhecimento científico e gerencial, assim a grade curricular do curso é multidisciplinar, com foco na solução de problemas numa óptica global.

\subsection{Evolução dos cursos em Engenharia de Produção}

Os primórdios da Engenharia de Produção remetem à Revolução Industrial, quando havia a necessidade de criação, difusão e gerenciamento de ambientes fabris, nos quais se iniciaram a implementação e o controle de métodos e técnicas padronizadas (BROADBERRY et al., 2013). Essa profissão surge na Inglaterra com a intitulação de Industrial Engineering, ou seja, Engenharia Industrial (PALMA et al., 2012). Posteriormente, a Engenharia de Produção aflui da Engenharia Industrial, visto que a primeira possui uma área de atuação maior, incluindo a área de serviços assim como a indústria manufatureira.

Em virtude da instalação de multinacionais no Brasil, bem como do desenvolvimento de indústrias nacionais, surgiu uma lacuna de gerenciamento e de engenharia nessas indústrias, que estavam se modelando, a exemplos mundiais, com a lógica taylorista. Nesse cenário, surge em 1958 na Escola Politécnica da Universidade de São Paulo (USP) o primeiro curso de Engenharia de Produção em território nacional, inicialmente como uma opção ao curso de Engenharia Mecânica (UNIVERSIDADE..., 2009).
Primariamente, na década de 1990, havia 15 cursos de Engenharia de Produção, enquanto em 2000 eram já 72 cursos, ou seja, houve um acréscimo na disponibilidade do curso de $1.820 \%$, crescimento muito superior ao das demais engenharias (BITTENCOURT et al., 2010). Nessa contagem, são inclusos todos os cursos de Engenharia de Produção plena, bem como os cursos com ênfase de Engenharia de Produção, tais como: Engenharia de Produção Mecânica, Engenharia de Produção Civil; Engenharia de Produção Agroindustrial; Engenharia de Produção e Qualidade; Engenharia de Produção e Sistemas; Engenharia de Produção Elétrica; Engenharia de Produção Química; Engenharia de Produção Eletromecânica, dentre outros (ASSOCIAÇÃO..., 2008).

O primórdio brasileiro nos cursos de pós-graduação em Engenharia de Produção foi no ano de 1967, com a implantação desses cursos na PUC-Rio e UFRJ (BITTENCOURT et al., 2010). O estado do Rio de Janeiro até hoje tem alta representatividade, pela quantidade de cursos em vigor em Engenharia de Produção, mantendo 53 cursos de graduação (ASSOCIAÇÃO..., 2013a), portanto, 10,31\% do total de cursos fornecidos no Brasil. Essa participação é ainda maior quando se trata do percentual da pós-graduação: $26,32 \%$ dos programas são disponibilizados naquele estado (ASSOCIAÇÃO..., 2013b).

A disponibilidade e crescimento tanto dos cursos de graduação como de pós-graduação em Engenharia de Produção ainda são ínfimos quando comparados com as necessidades do país, de acordo com Faé \& Ribeiro (2005): em termos médios, 7,7 vagas são ofertadas em Engenharia de Produção para cada milhão de PIB, ou, em termos populacionais, são ofertadas 54,2 vagas para cada milhão de habitantes. Tendo em vista que quanto mais bem qualificada a população, de maior potencialidade ela dispõe para o desenvolvimento da nação, essa lacuna profissional contribui para o perfil no qual a produção primária impera.

Nesse cenário, os cursos de Engenharia de Produção estão amadurecendo como um campo disciplinar, com a tendência de convergência dos cursos de Engenharia de Produção para a Engenharia de Produção plena (UNIVERSIDADE..., 2009). Nesse sentido, projeta-se para os próximos anos a ampliação de vagas universitárias, a unificação dos cursos em Engenharia de Produção plena, bem como o desafio de melhorar a qualidade de seu ensino, a fim de melhor responder às necessidades do mercado.

Complementarmente, Lappalainen (2009) entende que os graduados em engenharia estão enfrentando mudanças de requisitos em relação às suas competências, e que isso exige uma mudança concomitante dos currículos universitários, 
desafiando o sistema atual a fornecer aos graduandos habilidades relevantes que o mercado de trabalho exige atualmente.

No entanto, ainda, muitos cursos de engenharia têm no seu currículo o uso e a aplicação de teorias, equações e modelos em uma variedade de contextos de resolução de problemas, mas experiências de aprendizagem explícitas nos modelos e no processo de modelagem precisam ser incorporadas ao currículo, apresentando um desafio ao ensino da engenharia (CARBERRY \& MCKENNA, 2014).

Taguas et al. (2014) corroboram que a formação atual da futura geração de engenheiros deve trazer ferramentas e abordagens inovadoras ao ensino da engenharia, fornecendo elementos adequados para a tomada de decisão, justificadas com base no pensamento crítico e no desenvolvimento de habilidades.

Desse modo, o ensino superior tem que proporcionar uma educação voltada para a habilidade e o treinamento do aluno, colocando o futuro profissional em uma posição de destaque, tornando-o qualificado (ZHENG, 2011).

Por isso, outro ponto considerado importante por Ahn et al. (2014), apesar do recente interesse na área, é o estudo da liderança na engenharia, a fim de identificar as competências e características que os profissionais de engenharia que trabalham na indústria e na academia devem possuir.

Portanto, no entendimento de Pan et al. (2009), diferentemente da educação científica, o ensino de engenharia deve centrar-se na combinação entre a teoria e a prática e promover a capacidade de os alunos resolverem problemas práticos da engenharia, pois o objetivo é formar engenheiros.

\section{Metodologia}

Este estudo desenvolveu-se por meio de uma pesquisa de natureza qualitativa e descritiva no que se referem os objetivos, sendo que no plano de coleta dos dados foi desenvolvida por meio de uma pesquisa bibliográfica. Nesse sentido, Pizzinatto \& Farah (2012) afirmam que uma pesquisa qualitativa privilegia a obtenção de dados não quantitativos em profundidade sobre determinado tema de interesse e a busca de resultados a partir deles sem a pretensão de usar procedimentos estatísticos para sua análise.

A respeito do estudo descritivo, Cervo et al. (2007) explicam que esse tem como objetivo principal a descrição de algo visando à identificação, registro e análise das características, fatores e variáveis que se relacionam com o fenômeno ou processo estudado.

O estudo bibliográfico, por sua vez, tem por finalidade conhecer as diversas formas de contribuição científica existentes que foram realizadas sobre determinado assunto ou fenômeno, além de fornecer subsídios para o embasamento da pesquisa (JUNG,
2004). Malhotra et al. (2005) acrescentam que a pesquisa bibliográfica é caracterizada pela utilização de fontes secundárias, ou seja, quaisquer dados que já foram coletados para outros propósitos, podendo estar em pesquisas anteriores ou em documentos ou em publicações. Salienta-se que este trabalho utilizou também dados primários, isto é, aqueles levantados em primeira mão pelo pesquisador.

A pesquisa foi realizada entre os meses de abril de 2011 e agosto de 2013, com as seguintes três etapas, conforme a Figura 1: na primeira fase da pesquisa foi realizada coleta de dados secundários, por meio de levantamento documental e bibliográfico. Segundo Lakatos \& Marconi (2010), a pesquisa bibliográfica propicia o exame de um tema sob um novo enfoque ou abordagem, o que permite chegar a conclusões inovadoras, não sendo uma repetição do que foi anteriormente dito ou escrito sobre determinado assunto. Dessa forma, os dados têm como origem fontes secundárias que permitem a realização de estudos, de acordo com as aptidões do pesquisador que almeja elaborar um trabalho científico (LAKATOS \& MARCONI, 2010).

Como base de dados foram utilizados os sites da Associação Brasileira de Engenharia de Produção - ABEPRO (ASSOCIAÇÃO..., 2008), o e-MEC (BRASIL, 2013) e Capes (COORDENAÇÃO..., 2013). A organização dos dados foi realizada para contemplar o eixo do artigo e analisar os cursos brasileiros de graduação e pós-graduação em Engenharia de Produção considerando, especialmente, a situação geográfica e a classificação deles. Quanto ao tratamento e análise dos dados, esses se deram com base em uma leitura crítica do material coletado ao longo do período.

\section{Mapeamento dos cursos}

\subsection{Mapeamento dos cursos de graduação}

Atualmente são ofertados 531 cursos de graduação em Engenharia de Produção no Brasil. Desses, 17 se encontram em fase de fechamento ou suspensão e, por isso, não foram considerados na pesquisa. Dos cursos restantes (514), a maioria (457) são cursos de Engenharia de Produção plena (Figura 2).

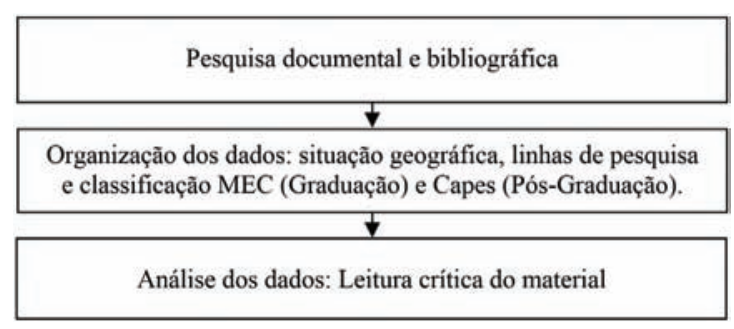

Figura 1. Mapa da pesquisa. Fonte: Elaborado pelos autores. 
Percebe-se, na Figura 2, uma grande concentração dos cursos de Engenharia de Produção nos estados de São Paulo (114), Minas Gerais (79) e Rio de Janeiro (51). Somente na cidade de São Paulo há 25 cursos de Engenharia de Produção, enquanto que no Rio de Janeiro são ofertados 20 cursos.

Além dos cursos de Engenharia de Produção plena, algumas universidades e faculdades fornecem cursos com ênfase em uma determinada área. $\mathrm{Na}$ Figura 3 estão dispostos, sob o mapa, os cursos de graduação em Engenharia de Produção.

Como previamente comentado, além dos cursos plenos em Engenharia de Produção existem 38 cursos de Engenharia de Produção Mecânica. Esses cursos concentram-se nos estados de São Paulo (26), Santa Catarina (5) e Amazonas (2). A cidade em destaque, novamente, é São Paulo, com sete cursos de graduação em Engenharia de Produção Mecânica. Um ponto atípico nesse cenário é a cidade de Manaus, com dois cursos.
Tal como a Engenharia de Produção Mecânica, em vigor há cursos de Engenharia de Produção com alguma ênfase (Figura 3) como:

a) Engenharia de Produção Civil: (oito cursos);

b) Engenharia de Produção Agroindustrial: (cinco cursos);

c) Engenharia de Produção e Qualidade: (um curso);

d) Engenharia de Produção e Sistemas: (um curso);

e) Engenharia de Produção Elétrica: (um curso);

f) Engenharia de Produção Química: (dois cursos); e

g) Engenharia de Produção Eletromecânica: (um curso).

Esses cursos com ênfase têm uma dominância nos estados do Paraná, Minas Gerais e Santa Catarina. Os cursos em Engenharia de Produção com ênfase em algum campo são uma minoria (57 cursos), ou seja, somente $11 \%$ dos cursos não estão na forma de Engenharia de Produção plena (Tabela 1).

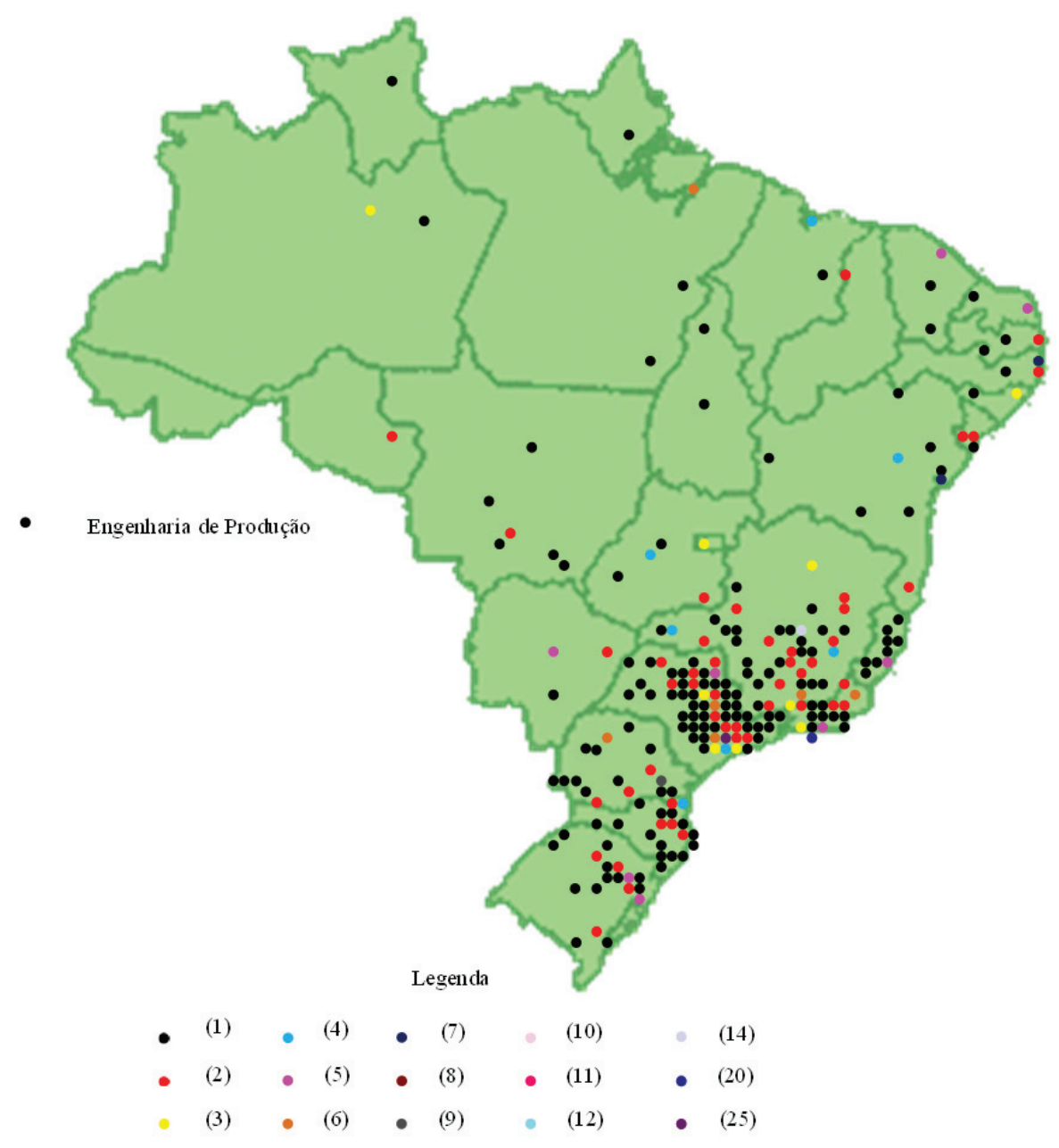

Figura 2. Mapa dos cursos de graduação em Engenharia de Produção. 


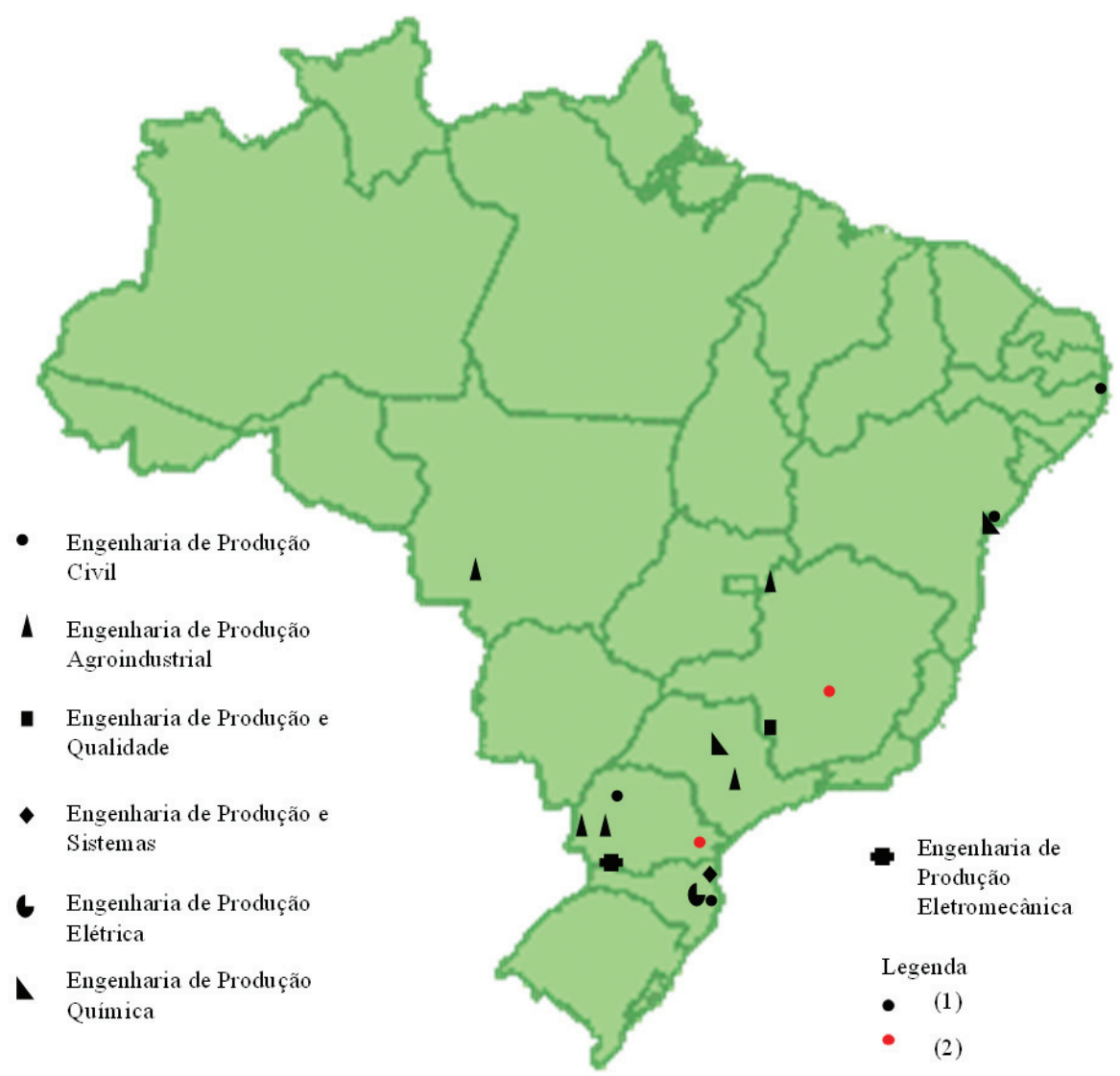

Figura 3. Mapa dos cursos de Graduação em Engenharia de Produção com diversas habilitações.

Tabela 1. Cursos de Graduação em Engenharia de Produção.

\begin{tabular}{lcc}
\hline \multicolumn{1}{c}{ Cursos } & Quantidade & Percentual (\%) \\
\hline Engenharia de Produção & 457 & 88,91 \\
Engenharia de Produção Mecânica & 38 & 7,39 \\
Engenharia de Produção Civil & 8 & 1,56 \\
Engenharia de Produção Agroindustrial & 5 & 0,97 \\
Engenharia de Produção e Qualidade & 1 & 0,19 \\
Engenharia de Produção e Sistemas & 1 & 0,19 \\
Engenharia de Produção Elétrica & 1 & 0,19 \\
Engenharia de Produção Química & 2 & 0,39 \\
Engenharia de Produção Eletromecânica & 1 & 0,19 \\
Total & $\mathbf{5 1 4}$ & $\mathbf{1 0 0 , 0 0}$ \\
\hline
\end{tabular}

Fonte: ABEPRO (ASSOCIAÇAO..., 2013) e Brasil (2013b).

Entre os cursos de Engenharia de Produção, além do curso pleno, se destaca o curso de graduação em Engenharia de Produção Mecânica, com presença de 7,39\%, conforme Tabela 1.

Salienta-se que os cursos de Graduação em Engenharia de Produção com alguma ênfase foram criados para atender demandas especiais que um profissional de Engenharia de Produção plena apresentaria maiores dificuldades para atender.

Para criar esses cursos se necessitam recursos maiores, já que além dos equipamentos e laboratórios de um curso pleno precisa-se criar toda estrutura para aquela habilitação. O que induz a que universidades privadas, com foco na rentabilidade, evitem esse investimento e prefiram disponibilizar o curso pleno de Engenharia de Produção.

Talvez isso explique, parcialmente, a tendência de diminuição da quantidade de cursos de Engenharia de Produção não plena oferecidos nos últimos anos, já que a grande maioria das universidades que oferecem cursos de Engenharia de Produção em habilitação são universidades públicas, ou seja, estaduais e federais. 


\subsection{Mapeamento dos cursos de pós-graduação}

No Brasil, existem, atualmente, 38 cursos de pós-graduação em Engenharia de Produção, classificados em mestrado acadêmico, mestrado profissional e doutorado. No caso dos Programas de Pós-Graduação em Engenharia de Produção, 38 instituições de ensino superior (privadas e públicas) possuem cursos dessa natureza (ASSOCIAÇÃO..., 2013b). Desses, nove são mais específicos em sua denominação, como: metrologia, logística e pesquisa operacional, metrologia científica e industrial, metrologia e qualidade, pesquisa operacional e inteligência computacional, planejamento energético, sistemas de gestão, sistemas e processos industriais e tecnologia, e os 29 restantes são intitulados como: Programa de Pós-Graduação em Engenharia de Produção ou em Engenharia de Produção e Sistemas.

A Figura 4 demonstra que há uma concentração de cursos de pós-graduação nos estados de São Paulo, Rio de Janeiro e Rio Grande do Sul. Na sequência, aparecem os estados do Paraná, Santa Catarina e Minas Gerais. Além desses, existem cursos de pós-graduação em Engenharia de Produção em sete outros estados, sendo que a maioria apresenta somente mestrado. A seguir, serão discutidas as linhas de pesquisa em cada curso de pós-graduação em Engenharia de Produção. Os 38 programas, com a especificação de mestrado acadêmico, mestrado profissional e doutorado, são apresentados na Tabela 1.

Os 38 programas de pós-graduação em Engenharia de Produção no Brasil têm no total 121 linhas de pesquisas distribuídas pelas 10 grandes áreas da Engenharia de Produção da ABEPRO (Tabela 2). A variação quanto à oferta dessas áreas é sensível, a área um, Engenharia de Operação e Processos da Produção, é a mais disponível, 24 cursos. Sequencialmente, na área oito, Engenharia Organizacional, é disponibilizada em 21 cursos.

A disponibilidade da pesquisa segue exposta com maiores detalhes na Tabela 2 .

A Tabela 2 apresenta que a Engenharia de Operações e Processos da Produção ( $1^{a}$ linha) é foco de pesquisa em 11 estados e abrange todo o Brasil. Já as pesquisas na segunda linha, Logística, têm predominância no estado São Paulo. A Pesquisa

- Mestrado

- Mestrado e Doutorado

- Mestrado Profissional, Mestrado e Doutorado

$\Delta \quad$ Mestrado Profissional

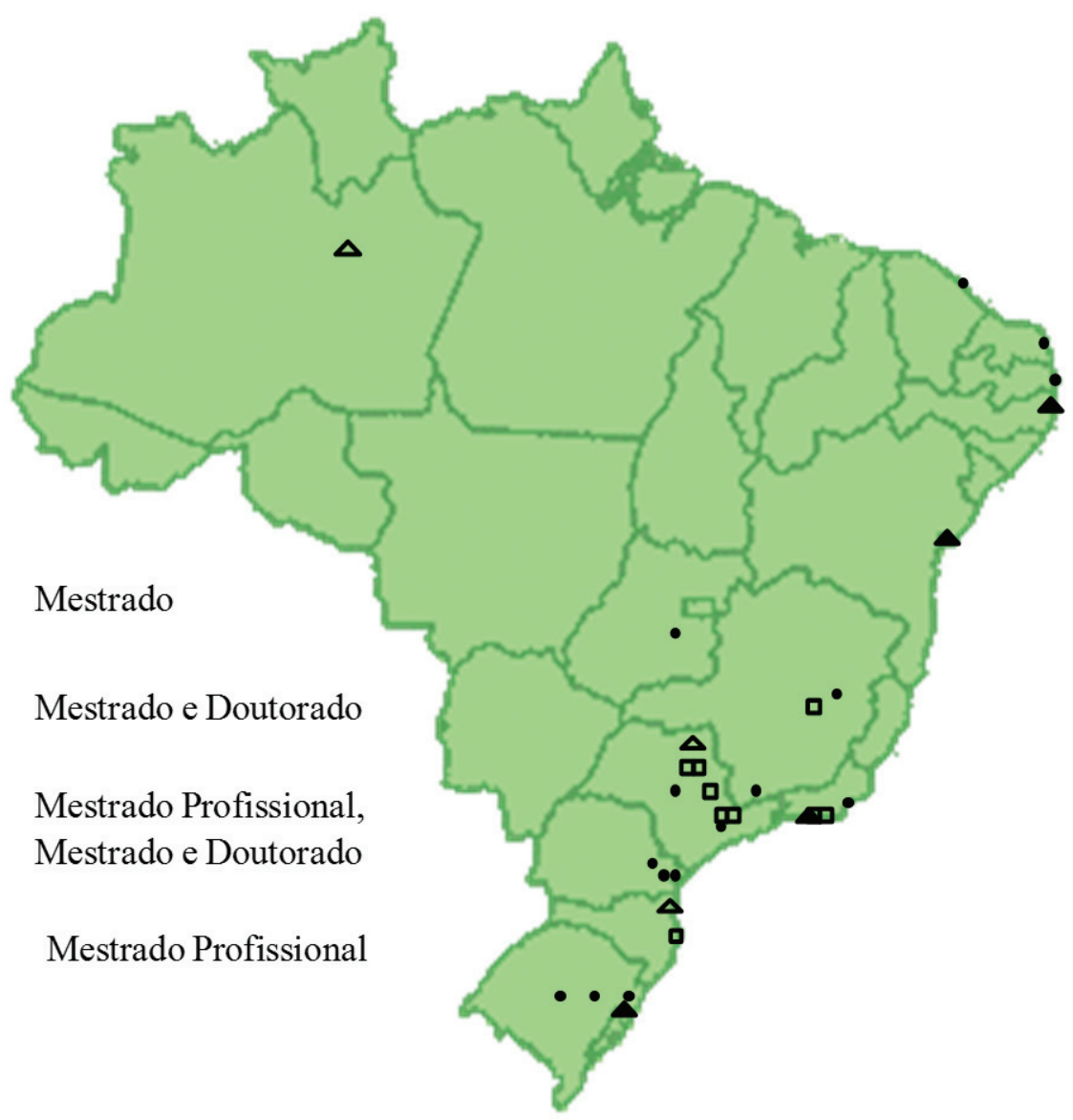

Figura 4. Cursos de pós-graduação em Engenharia de Produção. 
Tabela 2. Linhas de pesquisa na pós-graduação em Engenharia de Produção.

\begin{tabular}{|c|c|c|c|c|c|c|c|c|c|c|c|c|c|c|}
\hline \multirow[b]{2}{*}{ Linhas } & \multicolumn{3}{|c|}{ Sudeste } & \multicolumn{3}{|c|}{ Sul } & \multicolumn{5}{|c|}{ Nordeste } & \multirow{2}{*}{$\begin{array}{c}\begin{array}{c}\text { Centro- } \\
\text { Oeste }\end{array} \\
\text { GO } \\
\end{array}$} & \multirow{2}{*}{$\begin{array}{c}\text { Norte } \\
\text { AM } \\
\end{array}$} & \multirow{2}{*}{ Tota } \\
\hline & RJ & SP & MG & SC & PR & RS & BA & $\mathrm{CE}$ & PB & $\mathbf{P E}$ & $\mathbf{R N}$ & & & \\
\hline $\begin{array}{l}\text { 1. Engenharia } \\
\text { de Operações } \\
\text { e Processos da } \\
\text { Produção }\end{array}$ & 3 & 6 & 3 & 2 & 2 & 3 & 1 & 0 & 1 & 1 & 0 & 1 & 1 & 24 \\
\hline 2. Logística & 1 & 3 & 2 & 1 & 1 & 1 & 0 & 1 & 0 & 0 & 1 & 1 & 0 & 12 \\
\hline $\begin{array}{l}\text { 3. Pesquisa } \\
\text { Operacional }\end{array}$ & 4 & 3 & 2 & 1 & 0 & 2 & 0 & 0 & 0 & 0 & 1 & 0 & 1 & 14 \\
\hline $\begin{array}{l}\text { 4. Engenharia da } \\
\text { Qualidade }\end{array}$ & 3 & 1 & 0 & 0 & 1 & 0 & 0 & 0 & 0 & 0 & 0 & 0 & 1 & 6 \\
\hline $\begin{array}{l}\text { 5. Engenharia do } \\
\text { Produto }\end{array}$ & 1 & 2 & 1 & 1 & 1 & 1 & 0 & 0 & 1 & 0 & 1 & 0 & 0 & 9 \\
\hline $\begin{array}{l}\text { 6. Engenharia } \\
\text { Organizacional }\end{array}$ & 0 & 1 & 2 & 2 & 1 & 1 & 1 & 0 & 0 & 0 & 1 & 0 & 0 & 9 \\
\hline $\begin{array}{l}\text { 7. Engenharia } \\
\text { Econômica }\end{array}$ & 6 & 4 & 3 & 0 & 1 & 0 & 0 & 1 & 0 & 1 & 1 & 1 & 0 & 18 \\
\hline $\begin{array}{l}\text { 8. Engenharia do } \\
\text { Trabalho }\end{array}$ & 6 & 6 & 1 & 1 & 1 & 2 & 0 & 0 & 1 & 1 & 1 & 0 & 1 & 21 \\
\hline $\begin{array}{l}\text { 9. Engenharia da } \\
\text { Sustentabilidade }\end{array}$ & 2 & 3 & 1 & 0 & 1 & 0 & 0 & 0 & 0 & 0 & 1 & 0 & 1 & 9 \\
\hline $\begin{array}{l}\text { 10. Educação em } \\
\text { Engenharia de } \\
\text { Produção }\end{array}$ & 0 & 1 & 0 & 0 & 0 & 0 & 0 & 0 & 0 & 0 & 0 & 0 & 0 & 1 \\
\hline
\end{tabular}

Fonte: Adaptado de ABEPRO (ASSOCIAÇÃO..., 2013b).

Operacional se concentra nas regiões Sul, Sudeste, Centro-Oeste e Nordeste do Brasil, e a quarta linha (Engenharia da Qualidade) encontra-se basicamente nas regiões Sul, Sudeste e Norte. Os estados de Santa Catarina, Paraná, São Paulo e Minas Gerais apresentam dois cursos de pós-graduação que abordam a Engenharia do Produto como linha de pesquisa. Os estados do Rio Grande do Sul, Bahia e Rio Grande do Norte têm essa linha em apenas um curso. Já a Engenharia Organizacional é abordada em todos os programas de pós-graduação dos estados do Rio de Janeiro, Amazonas e, ainda, em alguns programas de pós-graduação nas regiões Sul, Sudeste, Centro-Oeste e Nordeste.

A Engenharia Econômica é uma linha pouco abordada, estando contemplada em seis programas, sendo que a metade deles se concentra no estado de Rio de Janeiro. Já a Engenharia do Trabalho é pesquisada em sete estados: Rio Grande do Sul, Santa Catarina, Paraná, São Paulo, Minas Gerais, Paraíba e Rio Grande do Norte. Uma linha relativamente nova é a Engenharia da Sustentabilidade. Ela é estudada em seis estados: Amazonas, Rio Grande do Norte, Minas Gerais, Rio de Janeiro, São Paulo e Paraná. A última linha da Engenharia de Produção é a Educação em Engenharia de Produção, que somente um programa de pós-graduação oferece.

\subsection{Linhas de pesquisas e relação entre os cursos de graduação e pós-graduação em Engenharia de Produção}

Considerando apenas os cursos cuja graduação atingiu em Engenharia de Produção o maior conceito nacional, CC igual a 5, e que também possuem mestrado e/ou doutorado ou mestrado profissionalizante em Engenharia de Produção, foram listadas as linhas de pesquisa em que essas instituições atuam (Tabela 3).

Na Tabela 3, inicialmente, é possível visualizar que, das sete instituições ditas de excelência, três estão localizadas no Sudeste, duas no Sul e duas no Nordeste. A quantidade de linhas de pesquisa variava consideravelmente, de duas, na UFC, para seis, na UNIMEP e na UFRGS. Salienta-se que o curso de Engenharia de Produção da UFRJ, apesar de possuir conceito Capes 6 na pós-graduação stricto senso, não foi incluso visto que a graduação dessa universidade atingiu apenas o conceito 4 no MEC.

Quanto às linhas pesquisadas, a linha 1, Engenharia de Operações e Processos de Produção obteve a maior pontuação e a linha 8, Engenharia do Trabalho, a maior proporção nos cursos (cinco), seguida pela linha 2, Logística, com pesquisa em quatro das sete universidades. Com pesquisa em três universidades têm-se: Pesquisa Operacional, Engenharia do Produto e Engenharia Econômica. 
Tabela 3. Linhas de pesquisas das universidades com pós-graduação cuja graduação atingiu maior conceito.

\begin{tabular}{|c|c|c|c|c|c|c|c|c|c|c|c|}
\hline $\begin{array}{ll} & \text { Linhas de pesquisa } \\
\text { Universidades }\end{array}$ & 1 & 2 & 3 & 4 & 5 & 6 & 7 & 8 & 9 & 10 & TOTAL \\
\hline UFF & 0 & 0 & 0 & 1 & 0 & 0 & 1 & 1 & 0 & 0 & 3 \\
\hline UFSCAR & 1 & 0 & 1 & 0 & 1 & 0 & 0 & 1 & 0 & 0 & 4 \\
\hline UNIMEP & 1 & 1 & 1 & 0 & 1 & 0 & 0 & 1 & 1 & 0 & 6 \\
\hline PUC-PR & 1 & 1 & 0 & 0 & 0 & 0 & 0 & 0 & 0 & 0 & 2 \\
\hline UFRGS & 1 & 1 & 1 & 0 & 1 & 1 & 0 & 1 & 0 & 0 & 6 \\
\hline UFC & 0 & 1 & 0 & 0 & 0 & 0 & 1 & 0 & 0 & 0 & 2 \\
\hline UFPE & 1 & 0 & 0 & 0 & 0 & 0 & 1 & 1 & 0 & 0 & 3 \\
\hline TOTAL & 5 & 4 & 3 & 1 & 3 & 1 & 3 & 5 & 1 & 0 & \\
\hline
\end{tabular}

Fonte: Adaptado de ABEPRO (ASSOCIAÇÃO..., 2013b).

Em apenas uma universidade têm-se: Engenharia da Qualidade, Engenharia Organizacional e Engenharia da Sustentabilidade. A linha 10, Educação em Engenharia de Produção não é pesquisada em nenhuma das universidades.

\section{Análise estatística}

\subsection{Análise estatística da graduação em Engenharia de Produção}

No levantamento foram considerados além dos dados gerais do curso de graduação em Engenharia de Produção também os indicadores como: Índice Geral de Cursos (IGC), Conceito Preliminar de Curso (CPC) e Conceito de Curso (CC).

Segundo o Instituto Nacional de Estudos e Pesquisas Educacionais Anísio Teixeira - INEP (INSTITUTO..., 2013), o IGC

é um indicador de qualidade de instituições de educação superior que considera, em sua composição, a qualidade dos cursos de graduação e de pós-graduação (mestrado e doutorado).

\section{Já o CPC}

é composto a partir dos resultados do Enade e por fatores que consideram a titulação dos professores, o percentual de docentes que cumprem regime parcial ou integral (não horistas), recursos didático-pedagógicos, infraestrutura e instalações físicas (BRASIL, 2013),

ou seja, ele é uma média de diferentes medidas da qualidade de um curso (Tabela 4).

O terceiro indicador da qualidade é o Conceito de Curso, que é calculado com base da avaliação in loco do curso pelo MEC (BRASIL, 2013). Assim, o $\mathrm{CC}$ pode confirmar ou modificar o CPC. O conceito dos três indicadores vai de 1 a 5 (sendo 5 o valor máximo).
Com base nos dados do e-MEC (BRASIL, 2013) foram calculados as médias para cada forma de curso de graduação em Engenharia de Produção, conforme a Tabela 4. Como foram utilizados somente cursos em andamento, então a média dos conceitos deve ser acima de 3.

Percebe-se na Tabela 4 que vários cursos são tão novos que ainda não têm um conceito do curso, como Engenharia de Produção e Sistemas, Engenharia de Produção Elétrica, Engenharia de Produção Química e Engenharia de Produção Eletromecânica. Em geral, pode-se dizer que os cursos em Engenharia de Produção plena têm um IGC menor mas recebem um Conceito do Curso maior. Somente o curso em Engenharia de Produção Agroindustrial consegue na média um CC maior, com um valor de 4,0. É possível visualizar também na referida tabela que os cursos de graduação em Engenharia de Produção que possuem mestrado e/ou doutorado ou mestrado profissionalizante em Engenharia de Produção apresentam média superior ao curso de Engenharia de Produção plena, Engenharia de Produção Civil e Engenharia de Produção Mecânica. Na Tabela 5, as variâncias em relação aos indicadores e modalidades são estimadas.

Verificando as variâncias para cada tipo de curso (Tabela 5), o curso em Engenharia de Produção pleno tem nos três indicadores as menores variâncias com valores de 0,3425 (IGC), 0,4711 (CPC) e 0,5040 (CC). As maiores variações existem no curso de Engenharia de Produção Mecânica e Agroindustrial no indicador CC, com valores de 1 . Os dois cursos de Engenharia de Produção Química têm o mesmo IGC, por isso têm uma variância de 1,0.

Na sequência, a Tabela 6 apresenta a correlação entre os indicadores e cursos - somente o curso em Engenharia de Produção Agroindustrial tem correlações mais significativas. Os demais cursos têm correlações bem fracas ou fracas, de forma positiva ou negativa.

Destaca-se a correlação forte entre o CPC e CC, com um valor de 0,8863 , que significa um $\mathrm{CPC}$ alto, que geralmente também causa um maior Conceito do 
Tabela 4. Média dos cursos de Engenharia de Produção.

\begin{tabular}{lccc}
\hline \multicolumn{1}{c}{ Cursos } & IGC & CPC & CC \\
\hline Engenharia de Produção & 3,1504 & 3,2205 & 3,7895 \\
Engenharia de Produção Mecânica & 3,5676 & 3,5200 & 3,5000 \\
Engenharia de Produção Civil & 3,6250 & 3,0000 & 3,0000 \\
Engenharia de Produção Agroindustrial & 3,6000 & 3,0000 & 4,0000 \\
Engenharia de Produção e Qualidade & 3,0000 & 3,0000 & 4,0000 \\
Engenharia de Produção e Sistemas & 4,0000 & 4,0000 & - \\
Engenharia de Produção Elétrica & 5,0000 & 4,0000 & - \\
Engenharia de Produção Química & 4,0000 & 3,0000 & - \\
Engenharia de Produção Eletromecânica & 4,0000 & - & - \\
Engenharia de Produção em Instituições com & 3,8065 & 3,6875 & $\mathbf{4 , 6 0 0}$ \\
mestrado/doutorado ou mestrado profissionalizante & & & $\mathbf{3 , 2 6 3 8}$ \\
Total & $\mathbf{3 , 2 0 6 3}$ & & $\mathbf{3 7 3 0}$ \\
\hline
\end{tabular}

Fonte: Dados da pesquisa.

Tabela 5. Variância dos cursos de Engenharia de Produção.

\begin{tabular}{lccc}
\hline \multicolumn{1}{c}{ Cursos } & IGC & CPC & CC \\
\hline Engenharia de Produção & 0,3425 & 0,4711 & 0,5054 \\
Engenharia de Produção Mecânica & 0,3535 & 0,4896 & 1,0500 \\
Engenharia de Produção Civil & 0,4844 & 0,5000 & 0,0000 \\
Engenharia de Produção Agroindustrial & 0,6400 & 0,6667 & 1,0000 \\
Engenharia de Produção e Qualidade & 0,0000 & 0,0000 & 0,0000 \\
Engenharia de Produção e Sistemas & 0,0000 & 0,0000 & - \\
Engenharia de Produção Elétrica & 0,0000 & 0,0000 & - \\
Engenharia de Produção Química & 1,0000 & 0,0000 & - \\
Engenharia de Produção Eletromecânica & 0,0000 & - & - \\
Engenharia de Produção em Instituições com mestrado/ & 0,4142 & 0,3398 & 0,4400 \\
doutorado ou mestrado profissionalizante & & & $\mathbf{0 , 4 8 8 7}$ \\
Total & $\mathbf{0 , 3 7 8 5}$ & & $\mathbf{0 , 5 4 3 1}$ \\
\hline
\end{tabular}

Fonte: Dados da pesquisa.

Tabela 6. Correlação dos índices dos cursos de Engenharia de Produção Agroindustrial.

\begin{tabular}{cccc}
\hline & IGC & CPC & CC \\
\hline IGC & 1 & & \\
CPC & 0,4063 & 1 & \\
CC & 0,6313 & 0,8863 & 1 \\
\hline
\end{tabular}

Fonte: Dados da pesquisa.

Curso. O contrário apresenta o curso em Engenharia de Produção Civil, onde a correlação entre o CPC e CC é fraca e negativa, ou seja, um curso que tem um Conceito Preliminar do Curso bom terá uma CC pior.

\subsection{Análise estatística da pós-graduação em Engenharia de Produção}

A média das linhas de pesquisa por programa de pós-graduação é 3,27, com um desvio padrão de 1,77. A correlação mede o grau de relacionamento entre duas variáveis (BARBETTA, 2005; MOORE, 2006). A correlação é representada pela letra $r$, cujo valor varia entre -1 e 1 (BANKHOFER \& VOGEL, 2008). Assim, os resultados possíveis são sem correlação $(r \approx 0)$; correlação positiva $(+r)$ (BARBETTA, 2005). Ocorre quando os valores de uma variável apresentam tendência crescente (ou decrescente) e os valores da outra variável possuem tendência igual à primeira; correlação negativa $(-r)$. Nesse caso, quando os valores de uma variável são crescentes (ou decrescentes), os valores da outra variável são decrescentes (ou crescentes). Para determinar o peso da correlação, utilizam-se os intervalos de valores entre 0 e 1 , conforme sugerido por Silva (2006).

A Tabela 7 apresenta as correlações entre os três programas de pós-graduação e entre as dez áreas da Engenharia de Produção. Percebe-se uma forte relação $(0,70)$ entre o mestrado e o doutorado. Assim, conclui-se que quando o mestrado é bem avaliado há uma forte tendência de o doutorado também o 
Tabela 7. Intervalos de valores da correlação.

\begin{tabular}{|c|c|c|c|c|c|c|c|c|c|c|c|c|c|}
\hline & M & D & MP & 1 & 2 & 3 & 4 & 5 & 6 & 7 & 8 & 9 & 10 \\
\hline Mestrado (M) & 1 & & & & & & & & & & & & \\
\hline Doutorado (D) & 0,70 & 1 & & & & & & & & & & & \\
\hline $\begin{array}{l}\text { Mestrado } \\
\text { profissional (MP) }\end{array}$ & $-0,34$ & 0,16 & 1 & & & & & & & & & & \\
\hline $\begin{array}{l}\text { 1. Engenharia } \\
\text { de Operações } \\
\text { e Processos da } \\
\text { Produção }\end{array}$ & 0,03 & 0,07 & 0,14 & 1 & & & & & & & & & \\
\hline 2. Logística & 0,31 & 0,27 & $-0,05$ & 0,17 & 1 & & & & & & & & \\
\hline $\begin{array}{l}\text { 3. Pesquisa } \\
\text { Operacional }\end{array}$ & $-0,06$ & 0,08 & 0,12 & $-0,10$ & 0,30 & 1 & & & & & & & \\
\hline $\begin{array}{l}\text { 4. Engenharia da } \\
\text { Qualidade }\end{array}$ & 0,17 & 0,30 & 0,04 & $-0,12$ & 0,02 & 0,12 & 1 & & & & & & \\
\hline $\begin{array}{l}\text { 5. Engenharia do } \\
\text { Produto }\end{array}$ & 0,06 & 0,15 & 0,03 & 0,17 & 0,29 & 0,34 & $-0,07$ & 1 & & & & & \\
\hline $\begin{array}{l}\text { 6. Engenharia } \\
\text { Organizacional }\end{array}$ & 0,10 & 0,18 & 0,18 & 0,17 & 0,42 & 0,22 & 0,10 & 0,42 & 1 & & & & \\
\hline $\begin{array}{l}\text { 7. Engenharia } \\
\text { Econômica }\end{array}$ & 0,18 & 0,05 & $-0,27$ & $-0,01$ & 0,11 & $-0,21$ & 0,07 & $-0,10$ & 0,03 & 1 & & & \\
\hline $\begin{array}{l}\text { 8. Engenharia do } \\
\text { Trabalho }\end{array}$ & 0,20 & 0,26 & $-0,02$ & $-0,03$ & $0,-04$ & 0,03 & 0,24 & 0,38 & 0,13 & 0,12 & 1 & & \\
\hline $\begin{array}{l}\text { 9. Engenharia da } \\
\text { Sustentabilidade }\end{array}$ & $-0,14$ & $-0,05$ & $-0,12$ & $-0,09$ & $-0,11$ & 0,09 & 0,10 & 0,27 & $-0,02$ & 0,15 & 0,00 & 1 & \\
\hline $\begin{array}{l}\text { 10. Educação em } \\
\text { Engenharia de } \\
\text { Produção }\end{array}$ & $-0,33$ & $-0,12$ & 0,20 & 0,13 & $-0,11$ & $-0,13$ & $-0,07$ & $-0,09$ & $-0,09$ & $-0,14$ & 0,15 & $-0,09$ & 1 \\
\hline
\end{tabular}

Fonte: Dados da pesquisa.

ser. Já entre o mestrado e o mestrado profissional a correlação é fraca $(-0,34)$. Ou seja, quanto melhor o mestrado, pior é o mestrado profissional. E entre o doutorado e o mestrado profissional é pior ainda, com valor de 0,16 . Dessa forma pode-se afirmar que o mestrado profissional não consegue se beneficiar da boa avaliação do doutorado.

As correlações entre as dez linhas de pesquisa apresentam-se, na maioria, bem fracas. Destaca-se a correlação entre a Engenharia do Produto e a Logística bem como entra a Engenharia do Produto e a Engenharia do Trabalho, que são moderadas, com valor de 0,42. A Engenharia da Qualidade e a Logística e a Engenharia da Qualidade e a Engenharia do Trabalho, bem como a Engenharia do Produto e a Engenharia Organizacional, fornecem correlações fracas, com valores: $0,30,0,34$ e 0,38, respectivamente.

\section{Considerações finais}

Os conhecimentos advindos da universidade influenciam as pessoas que, ao mesmo tempo, interrogam, buscam, cultivam e contestam o saber, as ciências, a tecnologia, as letras, as artes, assim como investigam e criam novos saberes e assumem a dimensão social e política da pesquisa, bem como se preparam para entrar no mercado de trabalho através das competências adquiridas no ambiente acadêmico.

Nesse contexto, com a realização deste estudo pode-se constatar que dentre as tendências dos cursos de Engenharia de Produção no Brasil, a convergência dos cursos de diversas ênfases para a Engenharia de Produção plena é um fenômeno considerável, visto que apenas $11 \%$ dos cursos de Engenharia de Produção possuem alguma ênfase, sendo que esses cursos estão dispostos principalmente nos estados do Paraná, Santa Catarina e Minas Gerais. Ainda que o governo tenha um grande impacto nesse sistema, considerando que esses cursos são disponibilizados na rede pública, o maior responsável por esse fenômeno, naturalmente, é o mercado. Isso se justifica pelo fato de o próprio mercado estar exigindo um profissional com uma determinada ênfase em uma região e de currículo mais generalista em outra.

Outro ponto relevante incide no fato de que as capitais dos estados de São Paulo e Rio de Janeiro, detentoras da maior incidência do curso pleno de Engenharia de Produção, possuem uma quantidade 
ínfima de cursos com ênfase em Engenharia de Produção (estado de São Paulo) ou nem possuem qualquer modalidade não plena (estado do Rio de Janeiro).

De modo generalista, destaca-se que a maior incidência dos cursos de Engenharia de Produção encontra-se, em ordem decrescente, nas regiões: Sudeste, Sul, Nordeste, Centro-Oeste e, por último, Norte. Contrapondo esse dado com o desenvolvimento industrial regional é possível afirmar que, à medida que o mercado regional possui maior necessidade do profissional de Engenharia de Produção, maior a disponibilização dos cursos de Engenharia de Produção tanto por faculdades particulares quanto por universidades públicas.

Quanto aos cursos de pós-graduação, a distribuição dos programas se concentra nos estados das regiões Sul e Sudeste, mais os estados de Goiás, Ceará, Paraíba, Rio Grande do Norte e Amazonas. Na distribuição das linhas de pesquisa, percebe-se grandes diferenças. A Educação em Engenharia de Produção é pesquisada em apenas uma universidade. Contudo, a linha de pesquisa de Engenharia de Operações e Processos da Produção, é pesquisada em 24 programas. A pesquisa da Engenharia Econômica se concentra no Estado Rio de Janeiro, onde ficam três dos seis programas de pós-graduação que apresentam esta linha.

$\mathrm{Na}$ verificação das correlações entre mestrado, doutorado e mestrado profissional percebe-se uma correlação forte $(0,70)$ entre mestrado e doutorado, fraca $(-0,34)$ entre mestrado e mestrado profissional e mais fraca ainda $(0,16)$ entre doutorado e mestrado profissional. Já, as correlações entre as linhas de pesquisa são muito fracas. Somente duas linhas de pesquisa conseguiram chegar a nivelar uma correlação moderada, são elas: Engenharia do Produto e Logística, bem como Engenharia do Produto e Engenharia do Trabalho. Além disso, três outras combinações atingiram correlação fraca.

A qualidade do ensino dessas instituições, tanto públicas quanto privadas, é avaliada pelo MEC para mantê-las em funcionamento. Nessa avaliação os cursos de Engenharia de Produção Civil apresentaram a menor média de CC, no limite do aceitável (3), as modalidades Engenharia de Produção Agroindustrial e Engenharia de Produção e Qualidade apresentaram média de (4), enquanto que a modalidade plena foi avaliada com um conceito de 3,79, aproximadamente. Dos três indicadores avaliados, a Engenharia de Produção plena apresentou a menor variância, situação esperada em virtude da dominância de quantidade de cursos da Engenharia de Produção plena frente às demais. Independente da modalidade do curso, o desafio dessas instituições de ensino e da sociedade é evidente: a educação necessita de uma evolução considerável.

Finalmente, percebe-se que apesar da existência de 38 programas de pós-graduação em Engenharia de Produção há ainda uma assimetria em relação às linhas de pesquisa, existindo grande potencial para ampliação bem como para aprofundamento da pós-graduação em Engenharia de Produção no Brasil.

Tendo em vista a análise previamente disposta, o presente artigo buscou contribuir para o conhecimento acerca dos cursos nacionais de Engenharia de Produção, considerando aspectos como a categoria de unidade de ensino, nomenclatura dos cursos e ênfase, bem como a situação geográfica. Por meio desta análise, é possível concluir que existe uma distribuição heterogênea de cursos, com uma concentração nas áreas industriais e economicamente mais bem desenvolvidas, principalmente nas regiões Sudeste e Sul, sendo que esses necessitam de uma reestruturação para atender com eficiência a real necessidade empresarial.

\section{Referências}

ASSOCIAÇÃO BRASILEIRA DE ENGENHARIA DE PRODUÇÃO - ABEPRO. Áreas e subáreas de Engenharia de Produção. Rio de Janeiro, 2008. Disponível em: <http://www.abepro.org.br/interna. asp?c=362>. Acesso em: 19 jul. 2013.

ASSOCIAÇÃO BRASILEIRA DE ENGENHARIA DE PRODUÇÃO - ABEPRO. Cursos de graduação. Rio de Janeiro. Disponível em: <http://www.abepro.org.br/ interna.asp? $\mathrm{p}=952 \& \mathrm{~m}=673 \& \mathrm{ss}=1 \& \mathrm{c}=399>$ >. Acesso em: 24 jul. 2013a.

ASSOCIAÇÃO BRASILEIRA DE ENGENHARIA DE PRODUÇÃO - ABEPRO. Cursos de Pós-Graduação. Disponível em: <http://www.abepro.org.br/interna. asp? $=952 \& m=674 \& s s=1 \& c=394>$. Acesso em: 16 ago. 2013b.

AHN, B. et al. Creating an instrument to measure leadership, change, and synthesis in engineering undergraduates. Journal of Engineering Education, v. 103, n. 1, p. 115-136, 2014. http://dx.doi.org/10.1002/ jee. 20036

BANKHOFER, U.; VOGEL, J. Datenanalyse und statistik. Wiesbaden: Gabler, 2008.

BARBETTA, P. A. Estatística aplicada às ciências sociais. 5. ed. Florianópolis: Editora UFSC, 2005.

BENFATTI, E. F. S. S.; STANO, R. C. M. T. Utilização da tecnologia em Educação a Distância na formação de engenheiros de produção da Universidade Federal de Itajubá: uma avaliação educacional. Gestão \& Produção, v. 17, n. 2, p. 433-446, 2010. http://dx.doi. org/10.1590/S0104-530X2010000200016

BITTENCOURT, H. R.; VIALI, L.; BELTRAME, E. A engenharia de produção no Brasil: um panorama dos cursos de graduação e pós-graduação. Revista de Ensino de Engenharia, v. 29, n. 1, p. 11-19, 
2010. http://dx.doi.org/10.15552/2236-0158/abenge. v29n1p11-19

BRASIL. Decreto $n^{\circ}$ 5.773. 2006. Diário Oficial da União, 10 maio 2006. Disponível em: <http://www. planalto.gov.br/ccivil_03/_Ato2004-2006/2006/ Decreto/D5773.htm\#art79>. Acesso em: 27 ago. 2013.

BRASIL. Ministério da Educação. Referenciais nacionais dos cursos de engenharia. Brasília. Disponível em: $<$ http://portal.mec.gov.br/dmdocuments/referenciais. pdf>. Acesso em: 26 set. 2014.

BRASIL. e-MEC. Busca interativa. Brasília, 2013. Disponível em: <http://emec.mec.gov.br/>. Acesso em: 24 jul. 2013.

BROADBERRY, S.; CAMPBELL, B. M. S.; LEEUWEN, B. When did Britain industrialise? The sectoral distribution of the labour force and labour productivity in Britain, 1381-1851. Explorations in Economic History, v. 50, n. 1, p. 16-27, 2013. http://dx.doi. org/10.1016/j.eeh.2012.08.004

CARBERRY, A. R.; MCKENNA, A. F. Exploring student conceptions of modeling and modeling uses in engineering design. Journal of Engineering Education, v. 103, n. 1, p. 77-91, 2014. http://dx.doi. org/10.1002/jee.20033

CERVO, A. L.; BERVIAN, P. A.; SILVA, R. Metodologia cientifica. 6. ed. São Paulo: Pearson Prentice Hall, 2007.

CHRYSSOLOURIS, G.; MAVRIKIOS, D.; MOURTZIS, D. Manufacturing Systems: Skills \&amp; Competencies for the Future. Procedia CIRP, v. 7, p. 17-24, 2013. http://dx.doi.org/10.1016/j.procir.2013.05.004

CONCEIÇÃO, I. S. S. et al. Histórico e situação atual do ensino de engenharia de produção no maranhão. In: ENCONTRO NACIONAL DE ENGENHARIA DE PRODUÇÃO, 32., 2012, Bento Gonçalves. Anais... Rio de Janeiro: ABEPRO, 2013. CD-ROM.

COORDENAÇÃO DE APERFEIÇOAMENTO DE PESSOAL DE NÍVEL SUPERIOR - CAPES. Brasília, 2013. Disponível em: <http://www.capes.gov.br/>. Acesso em: 24 jul. 2013.

DÍAZ BORDENAVE, J. E.; PEREIRA, A. M. Estratégias de ensino-aprendizagem. 32. ed. Petrópolis: Vozes, 2012.

FAÉ, C. S.; RIBEIRO, J. L. D. Um retrato da engenharia de produção no Brasil. Revista Gestão Industrial, v. 1, n. 3, p. 24-33, 2005. http://dx.doi.org/10.3895/ S1808-04482005000300003

FERRAZ, A. P. C. M.; BELHOT, R. V. Taxonomia de Bloom: revisão teórica e apresentação das adequações do instrumento para definição de objetivos instrucionais. Gestão \& Produção, v. 17, n. 2, p. 421-431, 2010. http://dx.doi.org/10.1590/S0104-530X2010000200015

FLEURY, A. O que é Engenharia de Produção? In: BATALHA, M. O. (Org.). Introdução à Engenharia de Produção. Rio de Janeiro: Elsevier, 2008.

FOLHA DE SÃO PAULO. Conheça os $\mathbf{1 5}$ profissionais mais procurados do país. São Paulo, 2013. Disponível em: <http://classificados.folha.uol.com.br/ especial/2013/procurados/>. Acesso em: 24 jul. 2013.

FOWLER, E. M.; MELLO, C. H. P.; COSTA NETO, P. L. O. Análise exploratória da utilização do programa de qualidade GESPÚBLICA nas instituições federais de ensino superior. Gestão \& Produção, v. 18, n. 4, p. 837-852, 2011. http://dx.doi.org/10.1590/ S0104-530X2011000400011

IAROZINSKI NETO, A.; LEITE, M. S. A abordagem sistêmica na pesquisa em Engenharia de Produção. Produção, v. 20, n. 1, p. 1-14, 2010. http://dx.doi. org/10.1590/S0103-65132010005000011

INSTITUTO NACIONAL DE ESTUDOS E PESQUISAS EDUCACIONAIS ANÍSIO TEIXEIRA - INEP. Indicador de qualidade das instituições de educação superior. Brasília. Disponível em: <http://portal.inep. gov.br/indice-geral-de-cursos $>$. Acesso em: 24 jul. 2013.

INSTITUTO DE PESQUISA ECONÔMICA APLICADA - IPEA. Radar: tecnologia, produção e comércio exterior. Brasília, 2011. Disponível em: <http://portal.mte.gov.br/data/files/8A7C816A2E7311 D1012FE444C1343095/IPEA_Radar12.pdf>. Acesso em: 19 jul. 2013.

JUNG, C. F. Metodologia para pesquisa e desenvolvimento: aplicada a novas tecnologias, produtos e processos. Rio de Janeiro: Axcel Books de Brasil, 2004.

LAKATOS, E. M.; MARCONI, M. A. Fundamentos de metodologia científica. 7. ed. São Paulo: Atlas, 2010.

LAPPALAINEN, P. Communication as part of the engineering skills set. European Journal of Engineering Education, v. 34, n. 2, p. 123-129, 2009. http://dx.doi.org/10.1080/03043790902752038

LOPES, E. M. T.; FARIA FILHO, L. M.; VEIGA, C. G. (Org.). 500 anos de educação no Brasil. 3. ed. Belo Horizonte: Autêntica, 2003.

MALHOTRA, N. K. et al. Introdução a pesquisa de marketing. São Paulo: Pearson Prentice Hall, 2005.

MOORE, D. Basic practice of statistics. 4. ed. West Lafayette: WH Freeman, 2006.

MOREIRA, M. A.; VEIT, E. A. Ensino superior: bases teóricas e metodológicas. São Paulo: EPU, 2010.

NAVEIRO, R. Saiba mais sobre Engenharia de produção. ABEPRO, 2000. Disponível em: <http://www.abepro. org.br/interna. asp? $\mathrm{p}=399 \& \mathrm{~m}=440 \& \mathrm{~s}=1 \& \mathrm{c}=417>$. Acesso em: 23 jul. 2013.

PALMA, M.; RÍOS, I.; GUERRERO, D. Higher education in industrial engineering in peru: towards a new model based on skills. Procedia: Social and Behavioral Sciences, v. 46, n. 1, p. 1570-1580, 2012.

PAN, X. et al. The function of production practical training in engineering education and teaching method reform. In: INTERNATIONAL CONFERENCE ON ENGINEERING EDUCATION, 2009, Malaysia. p. 7-8.

PASSOS, I.; VEIGA, A.; NAVES, M. L. P. (Org.). Currículo e avaliação na educação superior. Araraquara: Junqueira \& Marin, 2005.

PIZZINATTO, N. K.; FARAH, O. E. (Org.). Pesquisa pura e aplicada para marketing: processos e aplicações. São Paulo: Atlas, 2012.

ROCHA, R. P. Sentimentos de outono: sobre universidade e educação. Santa Maria: Ed. UFSM, 1997. 
SILVA, E. S. Correlação. Paraná, 2006. Disponível em: <http://leg.ufpr.br/ silvia/CE003/node74.html>. Acesso em: 27 ago. 2013.

SOUZA, P. N. P. LDB e educação superior: estrutura e funcionamento. 2. ed. São Paulo: Thomson Learning, 2001.

STALLIVIERI, L. Estratégias de internacionalização das universidades brasileiras. Caxias do Sul: Ed. UDUCS, 2004.

TAGUAS, E. V.; FALCONER, R.; TARQUIS, A. M. European Journal of Engineering Education, v. 39, n. 5, 463-466, 2014. http://dx.doi.org/10.1080/03043 797.2014.934323

UNIVERSIDADE FEDERAL DE SÃO CARLOS UFSCar. Projeto Pedagógico do Curso de Graduação em Engenharia de Produção da UFSCar: Campus São Carlos. São Carlos, 2009.
VELloso, J. (Org.). A pós-graduação no Brasil: formação e trabalho de mestres e doutores no país: direito, economia, engenharia mecânica, geociências, odontologia e psicologia. Brasília: Capes, 2003.

WEISE, A. D.; TRIERWEILlER, A. C. Comparação do ensino de engenharia de produção no Brasil e na Alemanha. Revista de Ensino de Engenharia, v. 29, n. 1, p. 29-39, 2010. http://dx.doi.org/10.15552/22360158/abenge.v29n1p29-39

ZHENG, C. Innovation and practice on collegeenterprise co-building for intramural productive practical training base of mould professional. In: PACIFIC-ASIA CONFERENCE ON CIRCUITS, COMMUNICATIONS AND SYSTEM (PACCS), 3., 2011, Wuhan. IEEE Transactions on Education, 2011. p. 1-2. 